\title{
Time resolution of cosmic noise observations with a correlation experiment
}

\author{
E. Nielsen ${ }^{1}$, F. Honary ${ }^{2}$, and M. Grill ${ }^{2}$ \\ ${ }^{1}$ Max Planck Institute for Aeronomy, 37191 Katlenburg-Lindau, Germany \\ ${ }^{2}$ Communications Research center, Faculty of Applied Sciences, Lancaster University, Lancaster, UK
}

Received: 31 July 2003 - Accepted: 20 November 2003 - Published: 8 April 2004

\begin{abstract}
A radio wave correlation experiment using a Mills Cross technique can be used to observe the cosmic noise intensity from the ground for the purpose of determining the absorption in the ionosphere. To ensure a time resolution of 10 to $20 \mathrm{~s}$ of the absorption observations, it is necessary that the autocorrelation function of the signal received within a wanted spatial resolution and in a given receiver is $>8 \%$ of the total signal amplitude incident on that receiver.
\end{abstract}

Key words. Radio Science (Instruments and techniques; Interferometry; Ionospheric physics)

\section{Introduction}

In riometer experiments the variations of the mean value of the cosmic noise received on the surface of the Earth is used to determine the absorption of radio waves in the ionosphere. Since the cosmic signal is a noise signal, its mean value can be determined only approximately. The larger the bandwidth of the receiver and the longer the time over which the signal is integrated, the more accurately the mean value is determined. The fluctuation of the mean value can be written as

$d A=10 \log \left(\frac{P_{o}}{P}\right)$,

where $P_{o}$ is the ideal mean value and $P$ is the current estimate. Let $P_{o}=P+d P$, where $\mathrm{dP}$ is the deviation of the actual mean value from $P_{o}$. Considering this deviation as noise on the measurements of $P$, one can define the Signal-to-Noise value of the measurements as $\mathrm{SNR}=P / d P$. Thus, the observed fluctuations of the cosmic noise intensity is expressed in $\mathrm{dB}$ in terms of the Signal-to-Noise of the measurements,

$d A=10 \log \left(1+\frac{1}{\mathrm{SNR}}\right)$.

Correspondence to: E. Nielsen

(nielsen@linmpi.mpg.de)
If a $d A<0.1 \mathrm{~dB}$ is required, then the observations of the cosmic noise intensity must be made with a SNR $>40$.

Riometer systems employ a single antenna or a group of antennas to detect the cosmic noise. In the case of a group of antennas the signals from each antenna are usually added together with an appropriate phase to provide the noise intensity in the antenna beam formed by the antenna group (see, for example, Detrick and Rosenberg, 1990). More recently, it was suggested to use a correlator experiment (Mills Cross technique) to form the antenna beams (Nielsen and Hagfors, 1997; hereafter NH97). The rationale behind this suggestion was to use a significant smaller number of antennas to achieve the same, or an improved, antenna beam width than would be required in an additive system. In this paper it is pointed out, that for a given accuracy, the integration time aimed at in NH97 may be overly optimistic (1 s). Considering that the correlation technique relies on extracting a small signal from within a very large signal, a realistic integration time may well be in excess of tens of seconds.

\section{Additive antenna arrays}

Using an antenna with an antenna lobe of some shape the fluctuations of the SNR of the cosmic noise received with that antenna is determined by the bandwidth, $B$, of the receiver used to detect the signal and by the integration time, $\tau$

$$
\mathrm{SNR}=\sqrt{\tau \times B} .
$$

The fluctuations on the absorption measurements one can obtain with this system is given by

$$
d A=10 \log \left(1+\frac{1}{\sqrt{\tau \times B}}\right) .
$$




\section{Correlation system}

A correlation system using a Mills Cross technique consists of two horizontal rows of antennas oriented perpendicular to each other. The antennas in each row are connected together with a linear phase progression along the row, to form a fan antenna lobe oriented perpendicular to the direction of the row and making an angle with the vertical that corresponds to the magnitude of the phase progression. Where two fan lobes (one for each row) intersect, a pencil antenna lobe is formed, and the intensity in the pencil lobe is determined by cross-correlating the signals received in the two fan lobes.

Thomson et al. (1991) determined the Signal-to-Noise ratio of the correlator output to be

$\mathrm{SNR}=\rho \sqrt{2 \times \tau \times B}$,

where $\rho(\ll 1)$ is the cross-correlation coefficient associated with the correlating signals in the pencil lobe. The factor " 2 " arises from a sampling rate that is twice the bandwidth.

It is assumed that the antenna gain in each fan is approximately constant as a function of direction inside the fan. The case is now considered in which each fan is subdivided into $N$ equally narrow angular intervals, each interval corresponding to one of the pencil lobes in which intensities are to be determined. Since all the cosmic noise intensity arriving within a particular antenna fan is detected on the receiver connected to this antenna fan lobe, only a fraction $1 / N$ of the total receiver signal is received within each of the smaller subdivisions corresponding to directional intensities. Outside the particular subdivision a fraction $(N-1) / N$ of the total signal is received. The signal received outside a particular subdivision is a signal for other subdivisions, but is considered noise for the particular subdivision. Thus, the fraction of the total signal received in any one fan lobe that is relevant to a particular subdivision can be approximated as

fraction $=\frac{\frac{1}{N}}{\frac{N-1}{N}}=\frac{1}{N-1}$.

Let "fraction" be an approximation of the relative amplitude of the signal received from a particular subdivision, or pencil antenna lobe, in comparison to the signal received in the whole fan. Then the cross-correlation value of the signal received in the pencil lobe is $\rho \sim 1 /(N-1)^{2}$.

The SNR of cosmic noise signal intensities received in a correlation system can now be written as

$\mathrm{SNR}=\frac{\sqrt{2 \times \tau \times B}}{(N-1)^{2}}$.

and the fluctuation on absorption measurements is, in $\mathrm{dB}$,

$d A=10 \log \left(1+\frac{(N-1)^{2}}{\sqrt{2 \times \tau \times B}}\right)$.

Compared with the case for additive antenna lobe formation, the fluctuating term in dA has increased by $\sim(N-1)^{2}$, where $N$ is the number of pencil lobes formed within each fan antenna lobe.

For a bandwidth of $1 \mathrm{MHz}$ and for SNR $>40$ we find a lower limit on the required integration time,

$\tau>8 \times 10^{-4} \times(N-1)^{4}$.

For SNR $\sim 40$ and variable $\mathrm{N}$, we find: for $N \leq 12, \tau \leq 12 \mathrm{~s}$, and for $N \leq 14, \tau \leq 23 \mathrm{~s}$. Thus, for a required accuracy on the absorption measurements of about $0.1 \mathrm{~dB}$, and an integration time in the range between 10 and $20 \mathrm{~s}$, a fan lobe should be subdivided into no more than about 12 pencil antenna lobes. This means that the power received in a pencil lobe should exceed $\sim 8 \%$ of the total power received in the fan lobe. Recent measurements with a correlation array tend to confirm these arguments (Grill et al., 2003).

Note that the performance of a Mills Cross antenna system is in accordance with theoretical predictions (see NH97), independent of the integration time. However, it is also clear that the antenna system may be designed to improve (reduce) the integration time for a given required accuracy.

In the following section some different configurations of antenna arrays are discussed with a view to improve the time resolution of the absorption measurements obtained with a Mills Cross technique.

\section{Antenna arrays}

The previous sections makes it clear that it is not the actual size of the antenna beams which determines the minimum usable integration time, but it is rather the size of the narrow beams relative to the width of the fan beam that is important. The narrow beam should be $>8 \%$ of the width of the fan beam. Thus, the slimmer the fan beam - the smaller the field of view - the narrower the narrow beams can be. The nature of the correlation technique when used to observe cosmic noise is such, that the better the spatial resolution, the smaller the field of view.

Nielsen (2001) described an antenna with 32 dipoles in each Mills Cross antenna row, separated by half a wavelength, and connected to a 32-element Butler matrix (see also NH97). Using linear tapering of the current amplitudes on the dipoles a radiation diagram for the correlation system was produced, with a side lobe performance equal to that of a $16 \times 16=256$ element square antenna array.

A comparable result could be achieved (without actually using tapering of the antenna currents) by combining the Butler matrix outputs pair wise into 16 outputs from the 32 element Butler matrix. The reason is that the combined outputs correspond to a cosine-tapering of the current amplitudes on the dipoles (Muenzer, 1972). It is to be evaluated what is technically easier: to taper the antenna signals, or to combine the Butler matrix outputs pair wise.

If we only consider lobes with a zenith angle less than 60 degrees, then for these cases we may use $\mathrm{N} \sim 14$. In order to further decrease $\mathrm{N}$, one would have to narrow the width of the fan beams, i.e. narrow the antenna beams in the direction 
perpendicular to the direction of the array arms. This implies that the antennas in the arms should have more narrow antenna diagrams than corresponds to a dipole. For example, one might use yagis instead of dipoles, or use two dipoles (separated by, say, 0.5 wavelength) instead of one, in effect widening the antenna arms.

Using antenna arms with 32-double dipoles connected in phase and the 32-antenna signals connected to a 32-element Butler matrix, and with the Butler matrix outputs connected pair wise, would constitute a system in which effectively $12 \times 12$ antenna lobes could be formed each lobe with a lobe-width of 7.6 degrees (corresponding to $12-\mathrm{km}$ horizontal resolution in the ionosphere), and the largest zenith angle $\sim 45$ degrees (corresponding to a spatial coverage of $180 \times 180 \mathrm{~km})$. The new system can achieve the same spatial resolution as a 16 by 16 additive field array antenna system, but with a reduced number of antennas which is a clear improvement.

Acknowledgements. Topical Editor M. Lester thanks A. Ranka for his help in evaluating this paper.

\section{References}

Detrick, D. L. and Rosenberg, T. J.: A Phased-array radio wave imager for cosmic noise absorption, Radio Sci., 25 (4), 325-338, 1990.

Grill, M., Honary, F., Nielsen, E., Hagfors, T., Dekoulis, G., Chapman, P., and Yamagishi, H.: A New Imaging Riometer based on Mills Cross Technique, in :7th International Symposium on Communication Theory and Applications, Ambleside, UK, 13th-18th July 2003, 26-31, 2003.

Hagfors, T.: Comparison of the performance of the Cross Correlation and the Filled Aperture Imaging Riometer, Lancaster University, 2001.

Muenzer, P. J.: Properties of linear phase arrays using Butler matrices, NTZ (Naturwisenschaftliche Technische Zeitschrift), Heft 9, 1972.

Nielsen, E.: Antenna system for a high resolution imaging riometer, MPAE-W-03-01-04, 2001.

Nielsen, E., Hagfors, T.: Plans for a new Rio-Imager Experiment in Northern Scandinavia, J. Atm. Solar-Terr. Phys., 59, 8, 939-949, 1997.

Thomson, A. R., Moran, J. M., and Swenson, Jr., G. W.: Interferometry and Synthesis in Radio Astronomy, Krieger Publishing Company, Malabar, Florida, 1991. 